\title{
Sur les propriétés des écoulements turbulents définis par des conditions aux frontières stationnaires
}

\author{
The properties of turbulent flows \\ defined by stationary boundary conditions
}

PAR A. FORTIER, PROFESSEVR A LA SORBONNE

\begin{abstract}
Les propriétés des éconlements turbulents définis par des conditions aux frontières stationnaires sont étudiées sur le cas particulier du problème de Couette en régime turbulent, puis généralisées à l'écoulement avec gradient de pression, à l'éconlement dans des conduits, et $\dot{a}$ la couche limite turbulente sur une plaque plane. Un certain nombre de résultats ont pu ètre établis, et concernent plus particulièrement la répartition des vitesses de l'écoulement moyen, ainsi que les lois de répartition des vitesses aux grands nombres de Reynolds.
\end{abstract}

\begin{abstract}
The properties of turbulent flows defined by stationary boundary conditions are studied with reference to the special case of Couette's problem in turbulent conditions. They are then generalised to apply to flow with a pressure gradient, flow in ducts, and the turbulent boundary layer along a flat plate. A certain number of results are established, more particularly in connection with the average flow velocity distribution and the relationship governing velocity distribution at high Reynolds numbers.
\end{abstract}

L'expérience montre qu'un écoulement défini par des conditions aux frontières stationnaires se présente toujours sous l'aspect turbulent, dans des régions plus ou moins étendues du domaine occupé par le fluide en mouvement, lorsqu'un ou plusieurs paramètres sans dimensions dépassent des valeurs critiques.

Nous nous limiterons au cas simple d'un fluide de masse volumique $\rho$ et de viscosité dynamique $\mu$ constantes. Nous supposerons que le champ des densités massiques des forces de volume dérive d'un potentiel et qu'il n'y a pas de surface frontière séparant deux fluides différents. Le seul paramètre sans dimension qui intervient dans ce cas est, comme on le sait, le nombre de Reynolds.

Pour des surfaces frontières de forme géométrique donnée, on peut définir plusieurs écoulements en imposant aux vitesses et aux contraintes, le long de ces surfaces, des conditions dynamiquement semblables, c'est-à-dire des conditions s'exprimant identiquement pour tous ces écoulements en choisissant convenablement les unités de longueur, masse et temps.

Ces unités convenables sont par exemple une longueur D caractérisant la grandeur des surfaces frontières, une masse $\mathrm{\rho D}^{3}$ et un temps que l'on peut toujours remplacer par une vitesse $\mathrm{V}$ en le multipliant par $D$. 
Les équations indéfinies des écoulements, comprenant les équations de Navier et l'équation de continuité, écrites avec ces unités, ne font apparaitre que le nombre de Reynolds $\mathscr{R}=\rho \mathrm{VD} / \mu$ qui dépend du problème particulier posé. Si donc on réalise pour tous ces écoulements l'égalité des nombres de Reynolds, les équations indéfinies et les conditions aux frontières sont identiques exprimées avec les unités $\mathrm{D}, \mathrm{pD}^{3}$ et $\mathrm{V}$. Par conséquent, si les conditions aux frontières introduites sont nécessaires et suffisantes pour définir une solution, il est bien évident que les solutions exprimées avec ces unités seront identiques pour tous les écoulements (on dira alors que les écoulements sont dynamiquement semblables).

Des difficultés théoriques apparaissent lorsqu'il s'agit de préciser la nature des conditions aux frontières nécessaires et suffisantes pour définir une solution, mais, dans tous les cas usuels, l'expérience permet de connaitre sans ambiguïé ces conditions et de dégager un certain nombre de propriétés générales des solutions.

Lorsque les conditions aux frontières sont rigoureusement indépendantes du temps et que le nombre de Reynolds est inférieur à une valeur critique, l'écoulement observé longtemps après le début du mouvement est rigoureusement stationnaire dans tout le domaine occupé par le fluide en mouvement. On dit que l'écoulement est partout laminaire stationnaire.

Pour certaines conditions aux frontières particulièrement simples, on sait alors trouver une solution des équations indéfinies. C'est le cas de l'écoulement de Stokes autour d'une sphère, de l'écoulement de Couette entre deux plans parallèles, de l'écoulement de Poiseuille dans un tube cylindrique de section circulaire ou plus généralement de section quelconque, de l'écoulement laminaire stationnaire dans la couche limite de la plaque plane, etc. Dans tous les cas que l'on sait traiter, la solution trouvée est en parfait accord avec l'expérience, ce qui confirme la validité des équations de Navier et des hypothèses sur la continuité des vitesses et des contraintes aux frontières, et en particulier de l'hypothèse d'adhérence du fluide sur les parois solides.

En fait, les équations de Navier contenant explicitement la variable temps, il faut, pour définir une solution, faire intervenir des conditions initiales. Dans les cas simples où on peut trouver une solution tenant compte des conditions initiales, on constate que cette solution tend vers une solution stationnaire, indépendante des conditions initiales, lorsque les conditions aux frontières sont devenues stationnaires depuis un temps suffisamment long, et l'expérience confirme parfaitement ce résultat.

Lorsque le nombre de Reynolds dépasse la valeur critique, il apparaît des régions où l'écoulement n'est plus stationnaire, bien que les conditions aux frontières soient maintenues rigoureusement indépendantes du temps. Pour des valeurs du nombre de Reynolds dépassant légèrement la valeur critique, l'écoulement est de type périodique, puis, pour des nombres de Reynolds plus grands, l'écoulement perd tout caractère de régularité; il devient aléatoire : on dit que l'écoulement dans les régions correspondantes est turbulent. Pour les grands nombres de Reynolds, on ne peut donc. plus parler d'une «solution» des équations indéfinies, puisqu'on en observe en réalité une infinité et, dans l'état actuel de nos connaissances, le problème ne peut plus être traité théoriquement.

Or, l'expérience montre que les écoulements turbulents définis par des conditions aux frontières rigoureusement stationnaires ont des propriétés générales analogues aux propriétés des écoulements laminaires stationnaires, mais ces propriétés ont un caractère statistique.

Pour préciser ce caractère, supposons qu'on effectue $N$ expériences avec des surfaces frontières de forme géométrique donnée et des conditions aux frontières stationnaires et dynamicuement semblables. L'expérience de rang $i$ est définic par les grandeurs $\rho_{i}, \mu_{i}, D_{i}, V_{i}$ el on choisit ces grandeurs de façon à réaliser l'égalité des nombres de Reynolds.

$$
\mathcal{R}_{i}=\frac{\rho_{i} V_{i} D_{i}}{\mu_{i}}
$$

On choisit par ailleurs, pour chaque expérience, un système d'axes de référence géométriquement lié aux surfaces frontières, de telle sorte que des points semblablement placés, que nous appellerons points homologues, par rapport aux surfaces frontières, aient des coordonnées proportionnelles à $D_{i}$. Dans ces conditions, si on effectue $N$ mesures à un instant quelconque d'une composante $u$ de la vitesse du fluide (suivant $O x$ par exemple) en des points homologues de chaque écoulement, on constate que la moyenne

$$
\frac{1}{N} \sum \frac{u_{i}}{V_{i}}
$$

est indépendante de $\mathrm{N}$, pourvu que ce nombre soit suffisamment grand. Ce résultat s'étend à toute moyenne portant sur les composantes de la vitesse et de leurs dérivées par rapport aux coordonnées 
et au temps, ainsi qu'aux moyennes portant sur les produits de ces grandeurs ou sur les composantes du tenseur des contraintes en des points homologues, à condition de choisir pour exprimer une grandeur quelconque mesurée dans l'écoulement de rang $i, \mathrm{D}_{i}$ comme unité de longueur, $\mathrm{o}_{i} \mathrm{D}_{i}{ }^{3}$ comme unité de masse et $V_{i}$ comme unité de vitesse.

Si au lieu d'effectuer $N$ mesures dans les $N$ écoulements, on effectue $p$ mesures dans le même écoulement à des instants successifs séparés par des intervalles de temps égaux, on constate que la moyenne de ces $p$ mesures est identique à la moyenne statistique définie à partir des $\mathrm{N}$ expériences distinctes, à condition que $p$ soit suffisamment grand.

Dans un écoulement turbulent défini par des conditions aux frontières stationnaires, les valeurs instantanées du vecteur vitesse et du tenseur des contraintes ne sont donc pas définies; seules les moyennes de ces grandeurs sont définies et, exprimées sous forme sans dimension, elle ne dépendent que du nombre de Reynolds. Comme dans les écoulements laminaires stationnaires, l'expérience montre que ces moyennes sont indépendantes des conditions initiales et par conséquent l'écoulement « moyen» ne dépend que des conditions aux frontières et d'un nombre de Reynolds au bout d'un temps suffisamment long après l'instant initial.

La similitude des propriétés des écoulements laminaires stationnaires et des écoulements turbulents stationnaires conduit à penser qu'il doit être possible de trouver dans les cas simples une solution «moyenne» des équations indéfinies satisfaisant à des conditions aux frontières données. Il faut pour cela être certain que les équations de Navier sont applicables aux écoulements turbulents. Or, on sait actuellement mesurer les vitesses et les taux de déformation instantanés dans un écoulement turbulent et on constate que ces taux de déformation sont en général bien inférieurs aux taux de déformation que l'on peut atteindre dans l'écoulement laminaire dans un tube capillaire, par exemple, c'est-à-dire dans des conditions telles que la formule de Poiseuille conséquence des équations de Navier, est vérifiée avec une très grande précision. Par ailleurs, il n'y a aucune raison de douter de la continuité des vitesses et des contraintes si on laisse de côté les écoulements de gaz aux très básses pressions. Nous admettrons donc la validité des équations de Navier et nous allons montrer sur l'exemple simple du problème de Couctte en régime turbulent (qui est en fait un schéma d'écoulements plus généraux) comment on peut établir un certain nombre de résultats, concernant ce type d'écoulement, à partir des hypothèses très générales que nous venons de préciser et de quelques hypothèses supplémentaires portant uniquement sur les caractères de symétrie de l'écoulement moyen : nous admettrons, comme on le fait couramment dans la recherche des solutions «laminaires», que l'écoulement moyen a la même symétrie que les conditions aux frontières.

\section{Ecoulement turbulent entre deux plans parallèles (problème de Couette)}

Soient deux plaques planes parallèles lisses s'étendant jusqu'à l'infini dans toutes les directions, telles que l'une des deux plaques soit fixe et l'autre animée d'un mouvement de translation uniforme de vitesse $\vec{V}$ parallèle aux deux plaques. Un fluide de masse volumique $p$ et de viscosité $\mu$ constantes remplit complètement l'espace compris entre les deux plaques.

Soit 2 e la distance des deux plaques, l'expérience montre que la structure de l'écoulement ne dépend que du nombre de Reynolds $\mathscr{R}=2 \mathrm{eV} / \nu(\nu=\mu / \rho)$ et pour des nombres de Reynolds supérieurs à une valeur critique de l'ordre de 1000 , l'écoulement entre les deux plaques est turbulent. L'écoulement moyen est du type plan et toutes les moyennes portant sur les composantes de la vitesse et leurs dérivées ne dépendent que d'une coordonnée comptée suivant un axe perpendiculaire aux deux plaques.

Prenons un axe $O x$ parallèle à $\vec{V}$ et de même sens situé sur la plaque fixe, un axe $O y$ perpendiculaire aux deux plaques et orienté positivement de la plaque fixe vers la plaque mobile et un axe $\mathrm{O} z$ perpendiculaire à $\mathrm{O} x$ et $\mathrm{O} y$. Soient $u, v, w$ les composantes de la vitesse du fluide en un point de coordonnées $x, y, z$ à l'instant $t$ suivant les trois axes.

Les hypothèses de base du calcul sont les suivantes:

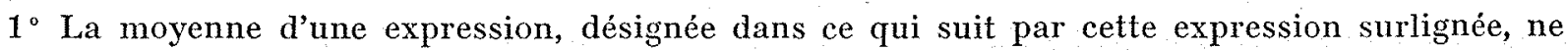
dépend que de $y$ et par conséquent on a, à titre d'exemple :

$\bar{u}=\frac{1}{t_{2}-t_{1}} \int_{t_{1}}^{t_{2}} u\left(x_{0}, y_{0}, z_{0}, t\right) d t=\frac{1}{x_{2}-x_{1}} \int_{x_{1}}^{x_{3}} u\left(x, y_{0}, z_{0}, t_{0}\right) d t=\frac{1}{z_{2}-z_{1}} \int_{z_{1}}^{x_{2}} u\left(x_{0}, y_{0}, z, t_{0}\right) d z$

à condition que $t_{2}-t_{1}$ soit grand devant $e / V$ et $x_{2}-x_{1}$ et $z_{2}-z_{1}$ grands devant $e$. 


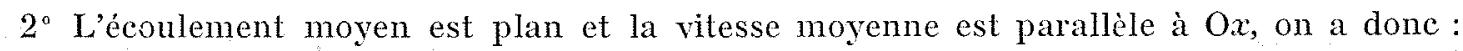

$$
\frac{\bar{u}}{\mathrm{~V}}=f\left(\mathfrak{R}, \frac{y}{e}\right) \quad \bar{v}=0 \quad \bar{w}=0
$$

$3^{\circ}$ L'écoulement moyen est isotrope dans la direction $\mathrm{O} z$, ce qui veut dire que les moyennes ne dépendent pas du choix de l'orientation de l'axe $\mathrm{O} z$; il en résulte que $\overline{u w}=0, \overline{v w}=0$. D'une facon plus générale, les moyennes telles que $\overline{u^{\alpha} v^{\beta} w^{\gamma}}$, appelées moments d'ordre $\alpha+\beta+\gamma$, sont nulles si $\gamma$ est impair et, si on choisit deux points $M_{1}$ et $M_{2}$ sur une parallèle à $O z$, tous les moments, moyennes des produits des composantes de la vitesse ou de leurs dérivées (autres que des dérivées par rapport à $z$ ) aux deux points $M_{1}$ et $M_{2}$, ne dépendent que de la distance $M_{1} M_{2}$.

$4^{\circ}$ Les moments des composantes de la vitesse ou de leurs dérivées en deux points $M_{1}$ et $M_{2}$ situés sur une parallèle à $\mathrm{O} x$ ou à $\mathrm{O} z$ sont tous nuls lorsque la distance $\mathrm{M}_{1} \mathrm{M}_{2}$ est grande devant $e$.

$5^{\circ}$ L'écoulement moyen est symétrique par rapport à un point quelconque $O^{\prime}$ d'ordonnée $y=e$. En effet, en rapportant le mouvement à des axes animés d'un mouvement de translation uniforme de vitesse $\vec{V} / 2$, les conditions aux frontières s'expriment symétriquement par rapport à $O^{\prime}$. En particulier, pour $y=e$, on a $\bar{u}=\mathrm{V} / 2$.

D'après les propriétés de symétrie, le tenseur des contraintes tangentielles moyennes sur les deux plaques se réduit à sa composante suivant $O x$. Nous désignerons par $\overline{\tau_{f}}$ la contrainte tangentielle moyenne exercée par le fluide sur la plaque fixe et nous poserons

$$
\frac{\overline{\tau_{0}}}{\rho \mathrm{V}^{2}}=\frac{c_{f}}{2}
$$

$c_{f}$ est le coefficient de frottement qui ne dépend, d'après nos hypothèses générales que du nombre de Reynolds $\mathcal{R}=2 \mathrm{eV} / \nu$.

Posons $u_{*}^{2}=\frac{\bar{\tau}_{0}}{\rho}$, c'est-à-dire $\left(\frac{u_{*}}{V}\right)^{2}=\frac{c_{f}}{2}$.

Le nombre de Reynolds $\mathcal{R}$ pouvant s'écrire $\mathcal{R}=2 \frac{e u_{*}}{\nu} \frac{\mathrm{V}}{u_{*}}$, le rapport $u_{*} / \mathrm{V}$ n'est fonction que du nombre de Reynolds $\mathfrak{R}_{*}=u_{*} e / \nu$ et par conséquent la relation $\frac{\bar{u}}{V}=f\left(\mathcal{R}, \frac{y}{e}\right)$ peut être mise sous la forme:

$$
\frac{\bar{u}}{u_{*}} \cdot \frac{u_{*}}{\mathrm{~V}}=f\left(\frac{2 e u_{*}}{v} \cdot \frac{\mathrm{V}}{u_{*}}, \frac{y u_{*}}{v} \cdot \frac{v}{u_{*} e}\right)
$$

c'est-à-dire :

$$
\frac{\bar{u}}{u_{*}}=f_{1}\left(\mathfrak{R}_{*}, \frac{y u_{*}}{\nu}\right)
$$

D’une façon plus générale, prenons $u_{*}$ comme unité de vitesse, $\nu / u_{*}$ comme unité de longueur et $\rho \nu^{3} / u^{3}{ }_{*}$ comme unité de masse (c'est-à-dire $\rho u^{2}{ }_{*}$ comme unité de pression et $\nu / u^{2}{ }_{*}$ comme unité de temps).

En affectant de l’indice + les variables sans dimensions exprimées à l'aide de ces unités, les équations indéfinies du mouvement s'écrivent

$$
\begin{gathered}
\frac{\partial u_{+}}{\partial t_{+}}+\frac{\partial u^{2}}{\partial x_{+}}+\frac{\partial u_{+} v_{+}}{\partial y_{+}}+\frac{\partial u_{+} w_{+}}{\partial z_{+}}=-\frac{\partial p_{+}}{\partial x_{+}}+\Delta u_{+} \\
\frac{\partial v_{+}}{\partial t_{+}}+\frac{\partial u_{+} v_{+}}{\partial x_{+}}+\frac{\partial v_{+}^{2}}{\partial y_{+}}+\frac{\partial v_{+} w_{+}}{\partial z_{+}}=-\frac{\partial p_{+}}{\partial y_{+}}+\Delta v_{+} \\
\frac{\partial w_{+}}{\partial t_{+}}+\frac{\partial u_{+} w_{+}}{\partial x_{+}}+\frac{\partial v_{+} w_{+}}{\partial y_{+}}+\frac{\partial w_{+}^{2}}{\partial z_{+}}=-\frac{\partial p_{-}}{\partial z_{+}}+\Delta w_{+} \\
\frac{\partial u_{+}}{\partial x_{+}}+\frac{\partial v_{+}}{\partial y_{+}}+\frac{\partial w_{+}}{\partial z_{+}}=0
\end{gathered}
$$


avec :

$$
\Delta=\frac{\partial^{2}}{\partial x_{+}^{2}}+\frac{\partial^{2}}{\partial y_{+}^{2}}+\frac{\partial^{2}}{\partial z_{+}^{2}}
$$

et les conditions aux frontières

$$
\begin{gathered}
u_{+}=v_{+}=w_{+}=0 \quad \text { pour } y_{+}=0 \\
u_{+}=\frac{\mathrm{v}}{u_{*}}=\mathrm{F}\left(\mathfrak{R}_{*}\right), v_{+}=w_{+}=0 \quad \text { pour } y_{+}=2 \mathfrak{R}_{*}
\end{gathered}
$$

D'après nos hypothèses générales, toutes les moyennes ne dépendent donc que de $y_{+}=y u_{*} / \nu$ et de $\mathcal{R}_{*}$ et d'après la définition de $u_{*}$ :

$$
\left(\frac{\partial \bar{u}_{+}}{\partial y_{+}}\right)_{y_{+}=0}=1
$$

\section{Propriétés de l'écoulement au voisinage des parois}

Nous admettrons, comme dans les écoulements laminaires, que $u_{+}, v_{+}, w_{+}, p_{+}$sont des fonctions continues et continuement dérivables des variables $x_{+}, y_{+}, z_{+}, t_{+}$; nous pouvons donc développer ces fonctions suivant les puissances croissantes de $y_{+}$au voisinage de $y_{+}=0$ et écrire

$$
\begin{aligned}
& u_{+}=a_{1} y_{+}+a_{2} \frac{y_{+}^{2}}{2}+\ldots+a_{n} \frac{y_{+}^{n}}{n !}+\ldots \\
& v_{+}=b_{1} y_{+}+b_{2} \frac{y_{+}^{2}}{2 !}+\ldots+b_{n} \frac{y_{+}^{n}}{n !}+\ldots \\
& w_{+}=c_{1} y_{+}+c_{2} \frac{y_{+}^{2}}{2 !}+\ldots+c_{n} \frac{y_{+}^{n}}{n !}+\ldots \\
& p_{+}=p_{+0}+d_{1} y_{+}+\ldots \ldots+d_{n} \frac{y_{+}^{n}}{n !}+\ldots
\end{aligned}
$$

$a_{i}, b_{i}, c_{i}, d_{i}$ qui désignent les dérivées partielles de $u_{+}, v_{+}, w_{+}$et $p_{+}$par rapport à $y_{+}$sont des fonctions continues et continuement dérivables des variables $x_{+}, z_{+}, t_{+}$.

D'après l'équation de continuité nous voyons que :

$$
b_{1}=0 \quad b_{n}=-\left(\frac{\partial a_{n-1}}{\partial \boldsymbol{x}_{+}}+\frac{\partial c_{n-1}}{\partial z_{+}}\right)
$$

Prenons les moyennes des deux membres des équations (1) (2) (3); nous obtenons, en tenant compte des propriétés des moyennes

$$
\begin{gathered}
\frac{\partial \overline{u_{+} v_{+}}}{\partial y_{+}}=-\frac{\partial \bar{p}_{+}}{\partial x_{+}}+\frac{\partial^{2} \bar{u}_{+}}{\partial y^{2}} \\
\frac{\partial \bar{v}_{+}^{2}}{\partial y_{+}}=-\frac{\partial \bar{p}_{+}}{\partial y_{+}} \\
0=-\frac{\partial \bar{p}_{+}}{\partial z_{+}}
\end{gathered}
$$

En dérivant l'équation (2') par rapport à $x_{+}$nous voyons que $\partial \overline{p_{+}} / \partial x_{+}$ne dépend pas de $y_{+}$ et par conséquent d'après l'équation (1') $\partial \overline{p_{+}} / \partial x_{+}$est une constante. Si on maintient à l'infini amont et à l'infini aval des pressions égales $\partial p_{+} / \partial x_{+}$est nul et les équations ( $\left.1^{\prime}\right)$ et ( $\left.2^{\prime}\right)$ deviennent :

$$
\begin{aligned}
& \frac{\partial \overline{u_{+}}}{\partial y_{+}}-\overline{u_{+} v_{+}}=1 \\
& \overline{p_{+}}+\overline{v_{+}{ }^{2}}=\text { Cte }
\end{aligned}
$$


Pour simplifier l'écriture nous supprimerons dans les calculs qui suivent les indices + lorsque aucune ambiguïté ne sera possible.

Portons les valeurs de $u$ et $w$ dans l'équation (2) et intégrons de $o$ à $y$. Nous obtenons :

$$
\begin{aligned}
p=p_{0} & +b_{2} y+b_{3} \frac{y^{2}}{2 !}+\left(b_{4}+\frac{\partial^{2} b_{2}}{\partial x^{2}}+\frac{\partial^{2} b_{2}}{\partial z^{2}}-\frac{\partial b_{2}}{\partial t}\right) \frac{y^{3}}{3 !} \\
& +\left(b_{5}+\frac{\partial^{2} b_{3}}{\partial x^{2}}+\frac{\partial^{2} b_{3}}{\partial z^{2}}-\frac{\partial b_{3}}{\partial t}-3 \frac{\partial\left(a_{1} b_{2}\right)}{\partial x}-6 b_{2}^{2}-3 \frac{\partial\left(c_{1} b_{2}\right)}{\partial z}\right) \frac{y^{4}}{4 !}+\ldots
\end{aligned}
$$

En remplaçant $p$ par son développement dans les équations (1) et (3) et en identifiant terme à terme nous obtenons finalement les relations

(5)

$$
\left\{\begin{array}{c}
\frac{\partial p_{0}}{\partial x}=a_{2} \\
5_{0} \\
5_{1} \\
\frac{\partial a_{1}}{\partial t}=2 \frac{\partial^{2} a_{1}}{\partial x^{2}}+\frac{\partial^{2} a_{1}}{\partial z^{2}}+\frac{\partial^{2} c_{1}}{\partial x \partial z}+a_{3} \\
5_{2} \frac{\partial a_{2}}{\partial t}+\frac{1}{2} \frac{\partial a_{1}^{2}}{\partial x}-3 a_{1} \frac{\partial c_{1}}{\partial z}+2 \frac{\partial\left(a_{1} c_{1}\right)}{\partial z}=2 \frac{\partial^{2} a_{2}}{\partial x^{2}}+\frac{\partial^{2} a_{2}}{\partial z^{2}}+\frac{\partial^{2} c_{2}}{\partial x \partial z}+a_{4} \\
\frac{\partial a_{3}}{\partial t}+2 \frac{\partial\left(a_{1} a_{2}\right)}{\partial x}-4 a_{1} \frac{\partial c_{2}}{\partial z}-2 a_{2} \frac{\partial a_{1}}{\partial x}-6 a_{2} \frac{\partial c_{1}}{\partial z}+3 \frac{\partial\left(a_{1} c_{2}+a_{2} c_{1}\right)}{\partial x}= \\
=2 \frac{\partial^{2} a_{3}}{\partial x^{2}}+\frac{\partial^{2} c_{3}}{\partial x \partial z}+\frac{\partial^{2} a_{3}}{\partial z^{2}}+\frac{\partial^{4} c_{1}}{\partial z \partial x^{3}}+\frac{\partial^{4} a_{1}}{\partial x^{4}}+\frac{\partial^{4} a_{1}}{\partial x^{2} \partial z^{2}}+ \\
+\frac{\partial^{4} c_{1}}{\partial x \partial z^{3}}-\frac{\partial^{3} a_{1}}{\partial x^{2} \partial z}-\frac{\partial^{3} c_{1}}{\partial x^{2} \partial \partial t}+a_{5}
\end{array}\right.
$$$$
(6)\left\{\begin{array}{cc}
6_{0} & \frac{\partial p_{0}}{\partial z}=c_{2} \\
6_{1} & \frac{\partial c_{1}}{\partial t}=2 \frac{\partial^{2} c_{1}}{\partial z^{2}}+\frac{\partial^{2} c_{1}}{\partial x^{2}}+\frac{\partial^{2} a_{1}}{\partial x \partial z}+c_{3} \\
6_{2} & \frac{\partial c_{2}}{\partial t}+\frac{1}{2} \frac{\partial c_{1}^{2}}{\partial z}-3 c_{1} \frac{\partial a_{1}}{\partial x}+2 \frac{\partial\left(a_{1} c_{1}\right)}{\partial x}=2 \frac{\partial^{2} c_{2}}{\partial z^{2}}+\frac{\partial^{2} c_{2}}{\partial x^{2}}+\frac{\partial^{2} a_{2}}{\partial x \partial z}+c_{4}
\end{array}\right.
$$

Si done on se donne les fonctions $a_{1}, a_{2}, c_{1}, c_{2}$ des variables $x, z$, les relations (5) et (6) permettent théoriquement de calculer tous les termes des développements de $u, v$, $w$, et $p$. Or, ces fonctions ne sont pas quelconques, car :

$$
\text { pour } y=2 \mathcal{R}_{*} \text { on doit avoir } u=\mathrm{V}, \quad v=0, \quad w=0
$$

le problème cst «fermé » par les conditions sur l'autre frontière.

On doit avoir par ailleurs $\overline{a_{1}}=1, \overline{a_{2}}=\overline{c_{1}}=\overline{c_{2}}=0$.

Sans chercher à calculer les moyennes et les moments non nuls des fonctions $a_{1}, a_{2}, c_{1}, c_{2}$ qui conduisent à des développements de $u, v, w$ satisfaisant aux conditions sur l'autre frontière, on peut établir un certain nombre de propriétés de l'écoulement moyen au voisinage de $y=0$ en partant des équations (5) et (6).

Comme beaucoup d'auteurs l'ont déjà remarqué, l'équation $\left(5_{1}\right)$ montre que $\bar{a}_{3}=0$. Le développement de $\bar{u}$ en fonction de $y$ ne contient donc pas de termes du deuxième et du troisième degré. Par ailleurs, puisque $b_{1}=0$, le développement de $v$ commence par un terme du deuxième degré en $y$ et, par conséquent, $v$ est infiniment petit par rapport à $u$ et $w$ lorsque $y$ tend vers zéro. Nous allons montrer qu'en fait, le premier terme non nul du développement de $\bar{u}$, après le terme $y$, est un terme du cinquième degré, car $\overline{a_{4}}=0$.

D’après l'équation $\left(5_{2}\right)$ nous avons :

$$
\overline{a_{4}}=-3 \overline{a_{1} \frac{\partial c_{1}}{\partial z}}
$$


Le moment $\overline{a_{1} \partial c_{1} / \partial z}$ n'est pas nul d'une façon évidente, car lorsqu'on change l'orientation de l'axe des $z, a_{1}\left(\partial c_{1} / \partial z\right)$ ne change pas de signe.

Intégrons l'équation $\left(5_{1}\right)$ de 0 à $z_{1}$ pour $x$ et $t$ donnés. Nous obtenons

$$
\begin{gathered}
\int_{0}^{\tilde{z}_{1}} \frac{\partial a_{1}}{\partial t} d z=2 \int_{0}^{z_{1}} \frac{\partial^{2} a_{1}}{\partial x^{2}} d z+\frac{\partial a_{1}\left(x, z_{1}, t\right)}{\partial z}-\frac{\partial a_{1}(x, o, t)}{\partial z}+\frac{\partial a_{1}(x, o, t)}{\partial x}-\frac{\partial c_{1}(x, o, l)}{\partial x}+ \\
+\int_{0}^{z} a_{3} d z
\end{gathered}
$$

Multiplions les deux membres de l'équation(7) par $c_{1}(x, o, t)$. Nous obtenons :

$$
\begin{gathered}
\int_{0}^{z_{1}} c_{1}(x, o, t) \frac{\partial a_{1}}{\partial z} d z=2 \int_{0}^{z_{1}} c_{1}(x, o, t) \frac{\partial^{2} a_{1}}{\partial x^{2}} d z+c_{1}(x, o, t) \frac{\partial a_{1}\left(x, z_{1}, t\right)}{\partial z}- \\
-c_{1}(x, o, t) \frac{\partial a_{1}(x, o, t)}{\partial z}+c_{1}(x, o, t) \frac{\partial c_{1}\left(x, z_{1}, t\right)}{\partial x}-\frac{1}{2} \frac{\partial c_{1}^{2}(x, o, t)}{\partial x}+ \\
+\int_{0}^{z_{1}} c_{1}(x, o, t) a_{3} d z
\end{gathered}
$$

Prenons finalement les moyennes dans le temps des deux membres de l'équation (8) en choisissant $z_{1}$ suffisamment grand pour que, conformément à nos hypothèses, les moments tels que $\overline{c_{1}(x, o, t) \frac{\partial a_{1}}{\partial z}\left(x, z_{1}, t\right)}$ soient nuls. Nous obtenons :

$$
\begin{gathered}
\int_{0}^{z_{1}} \overline{c_{1}(x, o, t) \frac{\partial a_{1}}{\partial t}(x, z, t)} d z=2 \int_{0}^{z_{1}} \overline{c_{1}(x, o, t) \frac{\partial^{2} a_{1}}{\partial x^{2}}(x, z, t)} d z- \\
-\overline{c_{1}(x, o, t) \frac{\partial a_{1}}{\partial z}(x, o, t)}+\int_{0}^{z_{1}} \overline{c_{1}(x, o, t) a_{3}(x, z, t)} d z
\end{gathered}
$$

Or, les moments tels que $\overline{c_{1}(x, o, t) \frac{\partial a_{1}}{\partial t}(x, z, t)}$ changent de signe lorsqu'on change l'orienta. tion de l'axe des $z$ et, comme ces moments ne doivent dépendre que de la distance des deux points, ils sont nécessairement nuls. Nous avons done :

$$
\overline{c_{1}(x, o, t) \frac{\partial a_{1}}{\partial z}(x, o, t)}=0
$$

et comme :

$$
\frac{\partial \overline{a_{1} c_{1}}}{\partial z}=0 \quad \overline{a_{1} \frac{\partial c_{1}}{\partial z}}=0
$$

et par suite :

$$
\overline{a_{4}}=0
$$

En partant de l'équation $\left(5_{1}\right)$, on démontre par la même méthode les relations :

$$
\begin{gathered}
\overline{\left(\frac{\partial c_{1}}{\partial x}\right)}=-\frac{\overline{\partial a_{1}}}{\partial z} \cdot \frac{\overline{\partial c_{1}}}{\partial x} \\
c_{2} \frac{\partial a_{1}}{\partial z}=-\overline{c_{2} \frac{\partial c_{1}}{\partial x}}=-\overline{a_{1} \frac{\partial c_{\dot{z}}}{\partial z}}
\end{gathered}
$$

et en partant de l'équation $\left(6_{1}\right)$,

$$
\begin{gathered}
\overline{\left(\frac{\partial a_{1}}{\partial x}\right)^{2}}=-2 \frac{\overline{\partial a_{1}}}{\partial x} \cdot \frac{\partial \overline{c_{1}}}{\partial z} \\
2 \overline{a_{2} \frac{\partial c_{1}}{\partial z}}=-\overline{a_{2} \frac{\partial a_{1}}{\partial x}}=-2 \overline{c_{1} \frac{\partial a_{2}}{\partial z}}
\end{gathered}
$$


Or :

$$
\frac{\overline{\partial a_{1}}}{\partial z} \cdot \overline{\frac{\partial c_{1}}{\partial x}}=\frac{\overline{\partial a_{1}}}{\partial x} \cdot \frac{\overline{\partial c_{1}}}{\partial z} \quad \text { car } \frac{\overline{\partial a_{1}\left(\partial c_{1} / \partial x\right)}}{\partial z}=\frac{\overline{\partial a_{1}\left(\partial c_{1} / \partial z\right)}}{\partial x}=0
$$

d'où :

$$
\left(\frac{\partial a_{1}}{\partial x}\right)^{2}=2\left(\frac{\partial c_{1}}{\partial x}\right)^{2}=-2 \frac{\partial \overline{a_{1}}}{\partial x} \cdot \frac{\partial c_{1}}{\partial z}
$$

Le même procédé de calcul appliqué aux équations $\left(5_{1}\right)$ et $\left(6_{1}\right)$ permet d'obtenir d'autres relations que nous n'écrirons pas, car nous les utiliserons pas ici, puis ce même procédé appliqué aux équations $\left(5_{2}\right)$ et $\left(6_{2}\right)$ conduit à des relations faisant intervenir des moments du troisième ordre et ainsi de suite.

Les relations obtenues à l'aide des équations $\left(5_{1}\right)$ et $\left(6_{1}\right)$ ont des conséquences importantes que nous allons examiner.

On peut tout d'abord à l'aide de ces relations calculer $\overline{b_{2}{ }^{2}}$. Nous avons en effet :

$$
\overline{b_{2}{ }^{2}}=\overline{\left(\frac{\partial a_{1}}{\partial x}+\frac{\partial c_{1}}{\partial z}\right)^{2}}=\overline{\left(\frac{\partial a_{1}}{\partial x}\right)^{2}}+\overline{\left(\frac{\partial c_{1}}{\partial z}\right)^{2}}+2 \frac{\partial a_{1}}{\partial x} \cdot \frac{\partial c_{1}}{\partial z}
$$

et par suite :

$$
\overline{b_{2}^{2}}=\overline{\left(\frac{\partial c_{1}}{\partial z}\right)^{2}}
$$

Or il est pratiquement impossible de mesurer la composante $v$ de la vitesse très près de la paroi. Il est par contre facile de mesurer la composante $w$ en disposant un fil chaud parallèlement à la vitesse moyenne ( $v$ étant négligeable devant $w$ très près de la paroi) * et à l'aide de deux fils chauds parallèles placés à la même distance $y_{0}$ de la paroi on peut déterminer le coefficient de corrélation $\overline{w\left(o, y_{0}, z, t\right) . w\left(o, y_{0}, o, t\right)} / \overline{w^{2}}$ en fonction de $z$ et déduire de ce coefficient de corrélation, par la méthode classique, $\left(\overline{\partial w / \partial z)^{2}}\right.$. En représentant les variations de $\sqrt{\left(\overline{\partial w / \partial z)^{2}}\right.}$ en fonction de $y$, on en déduit $\left(\overline{\left.\partial c_{1} / \partial z\right)^{2}}\right.$.

D'après des résultats de mesures, qui n'ont du reste pas été effectuées par la méthode que nous venons d'indiquer, $\overline{b_{2}{ }^{2}}$ doit être de l'ordre de $10^{-5}$. Pour $y_{+}=5, \sqrt{\overline{{b_{+}}^{2}}}$ est donc de l'ordre de quelques centièmes, alors que $\overline{u_{+}}$est égal à 5 et que $\sqrt{\overline{w_{+}{ }^{2}}}$ est de l'ordre de l'unité. On voit donc que jusqu'à $y_{+}=5, v$ est négligeable devant la composante de la vitesse parallèle à la paroi.

Par ailleurs $\overline{u_{+} v_{+}}=\bar{a}_{5} y^{4}+14 !+\ldots$ avec $\overline{a_{5}}=\overline{a_{2} \cdot \partial a_{1} / \partial x}-4 \overline{c_{2} \cdot \partial c_{1} / \partial x}$. Nous voyons done que le premier terme du développement de $\overline{u_{+} v_{+}}$est lié aux fluctuations du gradient de pression parallèlement à la paroi; la valeur absolue de $\overline{a_{5}}$ ne doit pas dépasser $1 / 1000$, de telle sorte que pour $y_{+}=5, \overline{u_{+} v_{+}}$est de l'ordre du centième, alors que $\overline{\partial u_{+}} / \partial y_{+}$est pratiquement égal à 1 . De $y_{+}=0$ à $y_{+}=5$, l'écoulement est donc assimilable à un écoulement laminaire fluctuant, la vitesse oscillant en grandeur et direction tout en restant parallèle à la paroi; la région correspondante est, comme on le sait, appelée «film laminaire».

On peut ensuite calculer les premiers termes des développements de $\overline{u_{+}{ }^{2}}, \overline{\omega_{+^{\prime}}{ }^{2}}$ en fonction de $y_{+}$. On a en effet :

$$
\begin{aligned}
& \overline{u_{+}{ }^{2}}=\overline{a_{1}^{2}} y_{+}{ }^{2}+\overline{a_{1} a_{2}} y_{+}{ }^{3}+\left(\frac{\overline{a_{2}^{2}}}{4}+\frac{\overline{a_{1} a_{3}}}{3}\right) y_{+}{ }^{4}+\ldots \\
& \overline{w_{+}{ }^{2}}=\overline{c_{1}^{2}} y_{+}{ }^{2}+\overline{c_{1} c_{2}} y_{+}{ }^{3}+\left(\frac{\overline{c_{2}{ }^{2}}}{4}+\frac{\overline{c_{1} c_{3}}}{3}\right) y_{+}{ }^{4}+\ldots
\end{aligned}
$$

et d'après les équations $\left(5_{1}\right)$ et $\left(6_{1}\right)$ :

$$
\begin{aligned}
& \overline{a_{1} a_{3}}=\frac{3}{2} \overline{\left(\frac{\partial a_{1}}{\partial x_{+}}\right)^{2}}+\overline{\left(\frac{\partial a_{1}}{\partial z_{+}}\right)^{2}} \\
& \overline{c_{1} c_{3}}=2 \overline{\left(\frac{\partial c_{1}}{\partial z_{+}}\right)^{2}}
\end{aligned}
$$

$* v$ est en fait négligeable en moyenne devant $w$. 
Or, l'expérience permet de calculer $\overline{\left(\partial a_{1} / \partial x_{+}\right)^{2}}$ et $\overline{\left(\partial c_{1} / \partial x_{+}\right)^{2}}$ d'après des résultats de mesures. Faisons en effet tourner les axes $\mathrm{O} x$ et $\mathrm{O} z$ de $+\pi / 4$ autour de $\mathrm{O} y$ et désignons par $\mathrm{O} x^{\prime}$ et $\mathrm{O} z^{\prime}$ les nouveaux axes et par $u^{\prime}$ et $w^{\prime}$ les projections de la vitesse sur $\mathrm{O} x^{\prime}, \mathrm{O} z^{\prime}$. Il est facile de voir que nous avons :

$$
\begin{aligned}
& \overline{\left(\frac{\partial u^{\prime}}{\partial x^{\prime}}\right)^{2}}=\overline{\left(\frac{\partial w^{\prime}}{\partial z^{\prime}}\right)^{2}}=\frac{1}{4}\left[\overline{\left(\frac{\partial u}{\partial x}\right)^{2}}+\overline{\left(\frac{\partial u}{\partial z}\right)^{2}}+\overline{\left(\frac{\partial w}{\partial x}\right)^{2}}+\overline{\left(\frac{\partial w}{\partial z}\right)^{2}}+4 \frac{\partial u}{\partial x} \cdot \frac{\partial w}{\partial z}\right] \\
& \overline{\left(\frac{\partial u^{\prime}}{\partial z^{\prime}}\right)^{2}}=\overline{\left(\frac{\partial w^{\prime}}{\partial x^{\prime}}\right)^{2}}=\frac{1}{4}\left[\overline{\left(\frac{\partial u}{\partial x}\right)^{2}}+\overline{\left(\frac{\partial u}{\partial z}\right)^{2}}+\overline{\left(\frac{\partial w}{\partial x}\right)^{2}}+\overline{\left(\frac{\partial w}{\partial z}\right)^{2}}-4 \frac{\overline{\partial u}}{\partial x} \cdot \frac{\partial w}{\partial z}\right] \\
& \overline{\left(\frac{\partial u^{\prime}}{\partial z^{\prime}}\right)^{2}}-\overline{\left(\frac{\partial u^{\prime}}{\partial x^{\prime}}\right)^{2}}=2 \overline{\frac{\partial u}{\partial x} \cdot \frac{\partial w}{\partial z}}
\end{aligned}
$$

$\left(\partial u^{\prime} / \partial x^{\prime}\right)^{2}$ et $\left(\partial u^{\prime} / \partial z^{\prime}\right)^{2}$ sont aisément mesurables au fil chaud à partir des coefficients de corrélation :

$$
\frac{\overline{u^{\prime}\left(o, y_{0}, o, t\right) u^{\prime}\left(0, y_{0}, z^{\prime}, t\right)}}{\overline{u^{\prime 2}}} \text { et } \frac{\overline{u^{\prime}\left(o, y_{0}, o, t\right) u^{\prime}\left(x^{\prime}, y_{0}, o, t\right)}}{\overline{u^{\prime 2}}}
$$

et on déduit de la relation (9) la valeur de $\overline{\left(\partial a_{1} / \partial x_{+}\right) \cdot\left(\partial c_{1} / \partial z_{+}\right)}$et par conséquent $\overline{\left(\partial a_{1} / \partial x_{+}\right)^{2}}$ et $\overline{\left(\partial c_{1} / \partial x_{+}\right)^{2}}$.

Remarquons que l'équation (9) combinée avec l'équation de continuité conduit, comme on le sait, à la relation :

$$
\overline{\left(\frac{\partial u}{\partial z}\right)^{2}}=2 \overline{\left(\frac{\partial u}{\partial x}\right)^{2}}
$$

lorsque la turbulènce est homogène et isotrope. Or, cette dernière relation n'est pas compatible avec la relation précédemment trouvée $\overline{\left(\partial a_{1} / \partial x_{+}\right)^{2}}=2 \overline{\left(\partial c_{1} / \partial x_{+}\right)^{2}}$ et, par conséquent, on n'a pas le droit de calculer la fonction de dissipation moyenne près de la paroi en utilisant la relation (10).

Il faut introduire le développement de cette fonction suivant les puissances croissantes de $y_{+}$.

L'expression générale de la fonction de dissipation moyenne:

$$
\begin{aligned}
\bar{\Phi}=\frac{\rho u_{*}^{4}}{\nu}\left[\overline{\left(\frac{\partial u_{+}}{\partial x_{+}}\right)^{2}}+\overline{\left(\frac{\partial u_{+}}{\partial y_{+}}\right)^{2}}+\overline{\left(\frac{\partial u_{+}}{\partial z_{+}}\right)^{2}}+\overline{\left(\frac{\partial v_{+}}{\partial x_{+}}\right)^{2}}+\overline{\left(\frac{\partial v_{+}}{\partial y_{+}}\right)^{2}}+\overline{\left(\frac{\partial v_{+}}{\partial z_{+}}\right)^{2}}+\overline{\left(\frac{\partial w_{+}}{\partial x_{+}}\right)^{2}}+\right. \\
\left.+\overline{\left(\frac{\partial w_{+}}{\partial y_{+}}\right)^{2}}+\overline{\left(\frac{\partial w_{+}}{\partial z_{+}}\right)^{2}}\right]
\end{aligned}
$$

devient en remplaçant $u_{+}, v_{+}, w_{+}$par leurs développements :

$$
\begin{aligned}
\bar{\Phi}=\frac{\rho u_{*}^{4}}{\nu}\left\{\overline{a_{1}^{2}}+\overline{c_{1}^{2}}+2 \overline{\left(a_{1} a_{2}\right.}+\overline{\left.c_{1} c_{2}\right)} y_{+}+\left[\overline{a_{2}^{2}}+\overline{b_{2}{ }^{2}}+\overline{c_{2}^{2}}+\overline{a_{1} a_{3}}+\overline{c_{1} c_{3}}+\overline{\left(\frac{\partial a_{1}}{\partial x_{+}}\right)^{2}+\overline{\left(\frac{\partial a_{1}}{\partial z_{+}}\right)^{2}}+}\right.\right. \\
\left.\left.+\overline{\left(\frac{\partial c_{1}}{\partial x_{+}}\right)^{2}}+\left(\frac{\partial c_{1}}{\partial z_{+}}\right)^{2}\right] y_{1}{ }^{2}+\cdots\right\}
\end{aligned}
$$

et en tenant compte des relations précédemment trouvées :

$$
\begin{array}{r}
\Phi=\frac{p u_{*}^{4}}{\nu}\left\{\overline{a_{1}^{2}}+\overline{c_{1}^{2}}+2\left(\overline{a_{1} a_{2}}+\overline{c_{1} c_{2}}\right) y_{+}+\left[\overline{a_{2}^{2}}+\overline{c_{2}{ }^{2}}+2 \overline{\left(\frac{\partial a_{1}}{\partial z_{+}}\right)^{2}}+4 \overline{\left(\frac{\partial c_{1}}{\partial z_{+}}\right)^{2}}+\right.\right. \\
\left.\left.+6 \overline{\left(\frac{\partial c_{1}}{\partial x_{+}}\right)^{2}}\right] y_{+}{ }^{2}+\ldots\right\}
\end{array}
$$

Les premiers termes du développement de $\bar{\Phi}$ peuvent être calculés à partir de mesures de $\overline{u_{+}^{2}}$ et $\overline{w^{2}+}$ en fonction de $y_{+}$et de mesures de :

$$
\overline{\left(\frac{\partial a_{1}}{\partial z_{+}}\right)^{2}}, \overline{\left(\frac{\partial c_{1}}{\partial z_{+}}\right)^{2}} \text { et } \overline{\left(\frac{\partial c_{1}}{\partial x_{+}}\right)^{2}}
$$

par les méthodes que nous avons indiquées. 
Les résultats expérimentaux que nous possédons actuellement montrent que $\overline{a_{1}{ }^{2}}=1,15$, $\overline{c_{1}^{2}}=0,01$ et que $\bar{\Phi}$ reste pratiquement constant dans le film laminaire et égal à $1,16 \rho u_{*}^{1} / \nu$.

\section{Propriétés de l'écoulement au voisinage de $y=e$}

Prenons des axes de référence parallèles aux axes utilisés dans le chapitre précédent, animés d'un mouvement de translation uniforme de vitesse $\vec{V} / 2$, et plaçons l'origine des coordonnées dans le plan de symétrie des deux plaques. Posons $\xi=x / e, \eta=(y / e)-1, \zeta=z / e, \tau=t u_{*} / e$. Prenons $u_{\text {* }}$ comme unité de vitesse, $e$ comme unité de longueur et $p e^{3}$ comme unité de masse; $u, v, w$ désignant les composantes de la vitesse suivant les anciens axes, désignons par $u_{d}$ la vitesse relative du fluide par rapport aux axes mobiles. Nous avons $u_{d}=u-\mathrm{V} / 2, u_{d+}=u_{+}-\mathrm{V}_{+} / 2$. Les équations indéfinies du mouvement du fluide s'écrivent :

$$
\begin{aligned}
& \frac{\partial u_{d+}}{\partial \tau}+ \frac{\partial u^{2}{ }_{d+}}{\partial \xi}+\frac{\partial u_{d+} v_{+}}{\partial \eta}+\frac{\partial u_{d++} w_{+}}{\partial \zeta}=-\frac{\partial p_{+}}{\partial \xi}+\frac{1}{\mathcal{R}_{*}} \Delta u_{d+} \\
& \frac{\partial v_{+}}{\partial \tau}+\frac{\partial u_{d+} v_{+}}{\partial \xi}+\frac{\partial v^{2}}{\partial \eta}+\frac{\partial v_{+} w_{+}}{\partial \zeta}=-\frac{\partial p_{+}}{\partial \eta}+\frac{1}{\mathfrak{R}_{*}} \Delta v_{+} \\
& \frac{\partial w_{+}}{\partial \tau}+\frac{\partial u_{d+} w_{+}}{\partial \xi}+\frac{\partial v_{+} w_{+}}{\partial \eta}+\frac{\partial w^{2}}{\partial \zeta}=-\frac{\partial p_{+}}{\partial \zeta}+\frac{1}{\mathcal{R}_{*}} \Delta w_{+} \\
& \frac{\partial u_{d+}}{\partial \xi}+\frac{\partial v_{+}}{\partial \eta}+\frac{\partial w_{+}}{\partial \zeta}=0
\end{aligned}
$$

et les conditions aux frontières :

$$
\begin{array}{ll}
\eta=0 & \overline{u_{d+}}=0 \\
\eta= \pm 1 & u_{d+}= \pm \mathrm{V}_{+} / 2, \quad v_{+}=0, \quad w_{+}=0
\end{array}
$$

La condition $u_{d+}= \pm \mathrm{V} / 2$ peut être remplacée par $\partial \overline{u_{d+}} / \partial \eta=\mathcal{R}$. pour $\eta= \pm 1$

Lorsque $\mathfrak{G}_{*}$ tend vers l'infini, la solution «moyenne» tend donc vers une solution indépendante de $\mathfrak{R}_{*}$. En particulier $\overline{u_{d+}}$ tend vers une fonction que nous désignerons par $g_{0}(\eta)$. Pour $\mathfrak{R}_{\text {。 }}$ très grand, on peut poser

$$
\overline{u_{d+}}=g_{0}(\eta)+\frac{g_{1}(\eta)}{a_{*}}
$$

en développant $\overline{u_{d+}}$ qui est une fonction de $\eta$ et de $\mathfrak{R}_{*}$, suivant les puissances croissantes de $1 / \mathfrak{R}_{\text {. }}$ et en limitant le développement aux deux premiers termes.

Si nous admettons que le développement de $\overline{u_{+}}$en fonction de $\overline{y_{+}}$doit se raccorder au développement de $\overline{u_{d+-}}$ en fonction de $\eta$, il faut nécessairement que :

$$
\overline{u_{+}}=f_{0}\left(y_{+}\right)+\frac{f_{1}\left(u_{-}\right)}{\mathfrak{R}_{*}}
$$

pour les grandes valeurs de $\mathfrak{R}_{*}, f_{1}\left(y_{+}\right) / \mathcal{R}_{*}$ tendant vers zéro pour une valeur donnée de $y_{+}$, lorsque $\mathcal{R} *$ tend vers l'infini.

Nous devons donc avoir, dans la région de raccordement:

$$
f_{0}\left(y_{+}\right)=g_{0}(\eta)+\frac{\mathrm{V}_{+0}}{2}
$$

lorsque $\mathfrak{R}_{*} \rightarrow \infty$.

On sait que l'identité fonctionnelle (9) conduit aux expressions:

$$
\begin{aligned}
f_{0}\left(y_{+}\right) & =\mathrm{A} \log y_{+}+\mathrm{B} \\
g_{0}(\eta) & =\mathrm{A} \log \eta+\mathrm{C} \\
\frac{\mathrm{V}_{+0}}{2} & =\mathrm{A} \log \mathfrak{R}_{*}+\mathrm{B}-\mathrm{C}
\end{aligned}
$$

valables en toute rigueur pour $\mathcal{R}_{*}$ infini, A, B, C, désignant des constantes. 
Lorsque $\mathcal{R}_{*}$ est très grand nous avons :

$$
f_{0}\left(y_{+}\right)+\frac{f_{1}\left(y_{+}\right)}{\mathfrak{R}_{*}}=g_{0}(\eta)+\frac{g_{1}(\eta)}{\mathfrak{R}_{*}}+\frac{V_{+0}}{2}+\frac{\mathrm{G}_{1}}{\mathfrak{R}_{*}}
$$

et par conséquent :

$$
\begin{aligned}
f_{1}\left(y_{+}\right) & =A_{1} \log y_{+}+B_{1} \\
g_{1}(\eta) & =A_{1} \log \eta+C_{1} \\
G_{1}\left(\mathfrak{R}_{*}\right) & =A_{1} \log \mathcal{A}_{*}+B_{1}-C_{1}
\end{aligned}
$$

d'où :

$$
\left\{\begin{array}{l}
\overline{u_{+}}=\mathrm{A}\left(1+\frac{\alpha}{\mathfrak{R}_{*}}\right) \log y_{+}+\mathrm{B}\left(1+\frac{\beta}{\mathfrak{R}_{*}}\right) \\
\overline{u_{a+}}=\mathrm{A}\left(1+\frac{\alpha}{\mathfrak{R}_{*}}\right) \log \eta+\mathrm{C}\left(1+\frac{\gamma}{\mathfrak{R}_{*}}\right) \\
\frac{\mathrm{V}_{ \pm}}{2}=\mathrm{A}\left(1+\frac{\alpha}{\mathfrak{R}_{*}}\right) \log \mathcal{R}_{*}+\mathrm{B}-\mathrm{C}+\frac{\mathrm{B} \beta-\mathrm{C}_{\Upsilon}}{\mathfrak{R}_{*}}
\end{array}\right.
$$

\section{Généralisation des résultats précédents}

Passons maintenant au cas de l'écoulement entre deux plaques parallèles lisses sous l'action d'un gradient de pression constant dans une direction parallèle aux deux plaques. Ce problème se traite exactement comme le problème de Couette et les propriétés de l'écoulement moyen au voisinage des parois sont identiques. Il faut simplement ajouter dans le développement de $\overline{u_{+}}$en fonction de $y_{+}$le terme $\overline{a_{2}} y^{2}+/ 2$ !, car dans ce cas $\overline{a_{2}}=\left(\partial \overline{p_{0}} / \partial x_{+}\right)=-\left(1 / \mathcal{R}_{*}\right)$ (le sens positif sur l'axe des $x$ étant le sens de l'écoulement moyen). Désignons par $\overline{u_{m}}$, la vitesse moyenne maximale dans le plan de symétrie des deux plaques et posons $u_{d}=u-\overline{u_{n}}$. Le développement de $\overline{u_{d+}}$ en fonction de $\eta$ est différent du développement trouvé dans le cas du problème de Couette, mais la fonction $\mathrm{f}_{0}\left(\mathrm{y}_{+}\right)$est la même, car les termes contenant $\overline{\alpha_{2}}$ s'accumulent lorsque $\mathcal{A}_{*}$ tend vers l'infini.

Le problème de l'écoulement turbulent établi dans un tube lisse de section circulaire de rayon $r_{0}$ se traite de la même façon en utilisant des coordonnées cylindriques et en posant $\mathcal{K}_{*}=r_{0} u_{*} / \nu$. En désignant par y la distance d'un point à la paroi comptée sur la normale à la paroi orientée positivement de la paroi vers le fluide, les équations du mouvement du fluide près de la paroi sont identiques aux équations du mouvement plan lorsque $y / r_{0}$ tend vers zéro. Or $y / r_{0}=y_{+} / \mathcal{R}_{*}$ et par conséquent $y / r_{0}$ tend vers zéro pour une valeur donnée de $y_{+}$lorsque $\boldsymbol{R}_{*}$ tend vers l'infini. La fonction $f_{0}\left(y_{+}\right)$est donc la même que dans le cas de l'écoulement plan.

D'une façon plus générale, on peut traiter le problème de l'écoulement turbulent établi dans un conduit cylindrique lisse de section quclconquc. Pour étudier le mouvement au voisinage de la paroi, on prend un système de coordonnées curvilignes : $x$ suivant une génératrice, $y$ suivant la normale à la paroi et $z$ suivant le contour de la section droite. Si le rayon de courbure du contour de la section droite n'est pas infiniment petit, l'écoulement moyen près de la paroi possède les mêmes propriétés que l'écoulement plan et la fonction $f_{0}\left(y_{+}\right)$est la même. Pour définir $y_{+}$il faut évidemment introduire la valeur locale de $\overline{\tau_{0}}$ qui varie dans le cas général le long du contour de la section droite. En dehors du voisinage de points singuliers du contour, pour lesquels le rayon de courbure est infiniment petit, $\bar{\gamma}_{\text {; }}$ et par conséquent $u_{*}$ varie très lentement en fonction de $z$; les propriétés d'isotropie de l'écoulement moyen dans la direction $\mathrm{O} z$ sont donc vérifiées, très près de la paroi et pour les grands nombres de Reynolds.

Examinons finalement le problème de l'écoulement turbulent dans la couche limite d'une plaque plane lisse. En prenant les mêmes axes que pour le problème de Couette et en désignant par $\delta$ l'épaisseur de la couche limite, on voit aisément que $\overline{\tau_{0}}$ et $\delta$ sont des fonctions de $x$. Pour étudier l'écoulement au voisinage d'un point de la paroi d'abscisse $x_{0}$ choisissons comme unité de vitesse la valeur de $u_{*}$ pour $x=x_{0}$; les équations indéfinies du mouvement sont les mêmes que dans le cas du problème de Couette et les propriétés basées sur l'isotropie de l'écoulement moyen dans la direction 
$\mathrm{O} z$ sont identiques. Mais les moyennes dépendent de $x$ et par conséquent les coefficients des termes du développement de $\overline{u_{+}}$en fonction de $y_{+}$ne sont pas identiques. On a par exemple :

$$
\overline{a_{4}}==\frac{1}{2} \frac{\partial \overline{a_{1}^{2}}}{\partial x_{+}}-3 \overline{a_{1}} \frac{\partial c_{1}}{\partial z_{+}}=\frac{1}{2} \frac{\partial \overline{a_{1}^{2}}}{\partial x_{+}}-\frac{3}{2} \frac{\partial \overline{c_{1}^{2}}}{\partial x_{+}}
$$

En posant $\mathfrak{R}_{*}=u_{*} \delta / \nu$, on peut montrer que les termes supplémentaires, qui s'introduisent par suite des variations des moyennes en fonction de $x$, tendent vers zéro lorsque $\mathcal{R}$. tend vers l'infini. La fonction $\overline{u_{+}}\left(y_{+}\right)$au voisinage de la paroi tend donc vers la fonction obtenue pour l'écoulement établi lorsque $\mathcal{R}$. tend vers l'infini.

Les lois universelles de répartition des vitesses, qui s'étendent du reste aux répartitions de toutes les moyennes non nulles, sont done des lois limites valables en toute rigueur pour un nombre de Reynolds infini. Pour les grands nombres de Reynolds, les expressions de ces lois doivent être complétées par des termes supplémentaires qui tendent vers zéro lorsque le nombre de Reynolds tend vers l'infini.

Par exemple, la loi logarithmique classique

$$
\overline{u_{+}}=\mathrm{A} \log y_{+}+\mathrm{B}
$$

valable pour des valeurs de $y_{+}$supérieures à 30 , doit s'écrire :

$$
\overline{u_{+}}=\mathrm{A}[1+\alpha(\boldsymbol{\alpha})] \log y+\mathrm{B}[1+\beta(\alpha)]
$$

les fonctions $\alpha(\mathfrak{R})$ et $\beta(\mathfrak{R})$ tendant vers zéro lorsque $\alpha$ tend vers l'infini. A et $B$ sont des constantes universelles mais les fonctions $\alpha(\mathcal{R})$ et $\beta(\mathcal{R})$ dépendent des conditions aux frontières loin de la paroi et par conséquent du problème particulier étudié.

Pour déterminer expérimentalenent les valeurs de $\alpha$ et $\beta$ il faut exécuter des mesures très précises, car, aux grands nombres de Reynolds, $\alpha$ et $\beta$ sont petits devant 1 . Ainsi pour un tube cylindrique de section circulaire, les très nombreux résultats expérimentaux que nous possédons se groupent bien autour de la courbe représentée par l'équation :

$$
u_{+}=5,65\left(1-\frac{3000}{\mathcal{R}}\right) \log _{10} y+5
$$

$\alpha$ désignant le nombre de Revnolds UD/ $v$ exprimé avec la vitesse $U$, quotient du débit par la surface de la section, et le diamètre $D$ de la section.

Ces résultats expérimontaux ne sont malheureusement pas assez précis pour permettre de mieux définir les valeurs de $\alpha(\mathfrak{R})$ et $B(\mathfrak{R})$, et nous donnons l'équation (10) simplement pour montrer que $\alpha(\mathcal{R})$ devient très petit devant 1 , lorsque $\mathcal{R}$ dépasse 100000 , mais n'est pas $d u$ tout négligeable pour des nombres de Reynolds de l'ordre de $10^{4}$. Or, beaucoup d'expériences ont été effectuées dans l'intervalle $10^{4}<\mathcal{K}<10^{5}$, c'est-à-dire pour des valeurs de $\mathcal{R}$ pour lesquelles $\approx$ ( $(\mathcal{K})$ et $3(\mathcal{R})$ ne sont pas négligeables devant 1 et dépendent comme nous l'avons vu du problème particulier posé. Par ailleurs, il est difficile de déterminer avec précision la pente de la droite représentant $u_{+}$en fonction de $\log y_{+}$, car la loi logarithmique n'est valable que dans un intervalle limité de valeurs de $\eta_{+}$, pratiquement entre 30 et $\Omega_{*} / 10$ pour un tube de section circulaire. Il n'est donc pas élonnant de constater une très grande dispersion entre les valeurs trouvées par divers expérimentateurs et ce n'est pas en groupant sur une mème figure tous les résultats connus que l'on pourra déduire du nuage de points obtenu la loi $u_{+}\left(\mathcal{R}, y_{+}\right)$. Il faut s'appuyer sur des considérations théoriques analogues à celles que nous avons développées, l'expérience fournissant des ordres de grandeur permettant de démarrer le calcul numérique et de le simplifier. 


\section{ABSTRACT \\ THE PROPERTIES OF TURBULENT FLOWS DEFINED BY STATIONARY BOUNDARY CONDITIONS}

This study deals with the turbulent flow of a fluid of constant density $e$ and dynamic viscosity $u$, the mass density field of the volumetric forces being derived from a potential, in the case when there is no boundary surface to separate two different fluids. The only non-dimensional parameter to be considered in this case is the Reynolds number.

The inference from the similar properties of stationary laminar and turbulent flows (i.e. flows defined by strictly stationary boundary conditions) is that it should be possible, in simple cases, to find an 'average' solution for the indefinite equations satisfying these boundary conditions.

Except in the case of gas flows under very low pressures, there seems to be little doubt that the Navier equations are valid. The author shows by a simple example that a certain number of results can be found from very general basic assumptions-which are discussed more closely in the introduction-and a few additional assumptions regarding the symmetry of the mean llow.

\section{Turbulent flow between two parallel plates (Couette's problem).}

The basic conditions of the problem are set out in the diagramm. The moving fluid fills the space between two infinitely long smooth flat parallel plates, one being fixed and the other moving at a constant velocity $\vec{V}$ with respect to the stationary one.

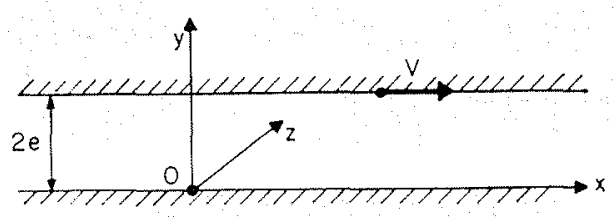

The basic calculation assumptions on the mean flow are: homogeneous isotropic flow in the direction $\mathrm{O} z$, and flow symmetry with respect to any point on the ordinate $y=e$. It is shown that the mean velocity distribution can be expressed by $u / u_{*}=f_{1}\left(\mathscr{R}_{*}, y u_{*} / v\right)$, and the indefinite equations of motion (1) to (4) are then written down, with $u_{*}, v / u_{*}$ and $Q v^{3} / u^{3}{ }_{*}$ respectively for the units of velocity, length and mass. Finally, the boundary conditions are defined.

\section{Properties of the flow near the walls.}

The author expands the functions $u_{+}, v_{+}, w_{+}$and $p_{+}$in the vicinity of $y_{+}=0$ and gives the form (1") and (2") obtained for the indefinite equations in this particular case by taking their mean value. Relations (5) and (6) are then established between the expansion coeflicients for the velocities and pressures, and a certain number of relationships are obtained in respect of their mean values, in particular the following:

$$
\begin{array}{ll}
\overline{a_{1}}=1 & \overline{a_{2}}=\overline{a_{3}}=\overline{a_{4}}=0 \\
\overline{b_{1}}=0 & \text { (also } b_{1}=0 \text { ) }
\end{array}
$$

It can be shown from certain measurement results that $\overline{b_{2}^{2}}$ is of the order of $10^{-5}$, from which the order of magnitude of $\sqrt{\overline{v^{2}}}$ (a few hundredths) can be calculated for $y_{+}=5$ and the nature of the flow in the 'laminar film' defined.

The same methods are also used to study the dissipation function. 


\section{Study of flow in the vicinity of $y=e$.}

Different basic units are considered in studying the flow in the median zone, i.e. $u_{*}, e$ and $\rho e^{3}$ for velocities, lengths and masses respectively.

After writing down the indefinite equations and boundary conditions, the author studies the form of the mean velocity distribution law at high Reynolds numbers and, by connecting it to the distribution law applicable near the walls, obtains the final relationships (12).

\section{Generalisation of the above results.}

Under this heading, the cases are considered of flow between two parallel plates with a constant pressure gradient in the direction $\mathrm{O} x$, of an established turbulent flow in a smoothwalled pipe of any cross-sectional area, and of the turbulent boundary layer along a smooth flat plate. The similarities and differences between these and the previous case are brought into evidence.

The author concludes with a few considerations on universally applicable velocity distribution laws.

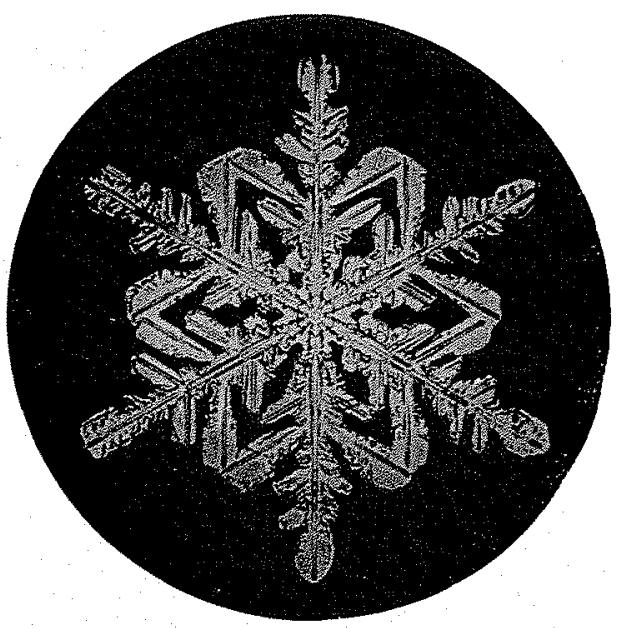

